Dear author,

Please note that changes made in the online proofing system will be added to the article before publication but are not reflected in this PDF.

We also ask that this file not be used for submitting corrections. 


\section{Trends in Biotechnology}

\section{CellPress \\ REVIEWS}

1

Q2

3 Antimicrobial Inks:

4 The Anti-Infective

5 Applications of

6 Bioprinted Bacterial

7 Polysaccharides

Q4 Q3 Ronan R. McCarthy, ${ }^{1, *, @}$

9 Muhammad Wajid Ullah, ${ }^{2}$

10 Eujin Pei, ${ }^{3}$ and Guang Yang ${ }^{2}$

11

12

13

14

15

16

17

18

19

20

21

22

23

24

25

26

27
Bioprinting is a rapidly emerging technology with the potential to transform the biomedical sector. Here, we discuss how a range of bacterial polysaccharides with antibiofilm and antibacterial activity could be used to augment current bioink formulations to improve their biocompatibility and tackle the spread of antibiotic-resistant infections.

\section{Printing Bacteria and Bacterial Polysaccharides}

Additive manufacturing or 3D printing has spearheaded a revolution in the biomedical sector for rapidly prototyping medical devices and personalized therapeutic solutions. The field of $3 \mathrm{D}$ bioprinting stemmed from the idea of combining $3 \mathrm{D}$ printing, which uses layer-by-layer fabrication techniques, with living organisms and biomaterials to produce complex tissues in vitro. Bioprinting can be classified into four main process categories: material jetting, laser-assisted printing, stereolithography, and material extrusion. Material jetting is a droplet-based technique that provides a high-throughput method with the ability to precisely control the displacement of biological material. It is compatible with a range of hydrogel formulations, including alginate, agarose, collagen, and fibrinogen [1]. Laserassisted printing uses laser-induced forward transfer to pattern cells over a given surface. This allows the positioning of small volumes of cell suspensions with high-resolution accuracy. This technology has been used to print a range of cell types, including embryonic stem cells, with a limited impact on cell viability. Stereolithography is a modified form of laser-assisted printing that uses an energy source, usually through laser curing or ultraviolet (UV) light to selectively initiate the polymerization process within a vat containing the photosensitive polymer, such as polyethylene glycol-diacrylate (PEGDA) and gelatin methacryloy [2]. Material extrusion is the most commonly used method of bioprinting. It utilizes physical forces, such as pneumatic pressure, to force a bioink through an extrusion nozzle and deposit it on a surface substrate in a coordinated fashion. Applications, including bone, tendon, skin, cardiovascular, and other types of tissue engineering, can be realized using material extrusion processes. In addition, extrusion processes enable adjustable pressure settings to accommodate the processing of materials with a range of viscosities [2]. Such recent advances in bioprinting have significantly affected the development of potentially new applications for tissue engineering and regenerative medicine (Figure 1).

Bacterial polysaccharides have emerged as a key component of many of the inks used in bioprinting [3]. These bacterial polysaccharides can influence key features, such as the mechanical and thermal properties, printability, biocompatibility, and biodegradability. However, implanting any foreign structure in the body comes with an increased risk of bacterial infection and, in particular, bacterial colonization of the implant itself [4]. Pathogenic bacteria can form communities called biofilms on these implanted structures and, when growing in a biofilm, bacteria are more tolerant to the rigors of the host immune system and antimicrobial therapy. Most hospital-related bacterial infections involve biofilm formation, with bacteria attaching 47 to implanted foreign objects, such as 48 prosthetic joints, dental implants, cathe- 49 ters, or intravenous lines, being a leading 50 cause of morbidity [4]. Integrating bacterial 51 polysaccharides with native anti-infective 52 properties into bioink formulations can 53 reduce the risk of infection and have a 54 role in removing a key barrier to the further 55 uptake of 3D bioprinting technology within 56 the biomedical sector. Anti-infective poly- 57 saccharides can also ease some of the 58 pressure on the healthcare system caused 59 by antibiotic-resistant infections.

60

Bioactive Bacterial

61

Polysaccharides

62

Bacteria are rich reservoirs for polysac- 63 charides and, while the primary use of 64 bacterial polysaccharides in bioprinting is 65 to confer structural properties [3], many 66 have been shown to have secondary func- 67 tionalities. An increasingly diverse array of 68 bacterial polysaccharides has been identi- 69 fied that display antibiofilm activity both 70 in vitro and in vivo. The functional capacity 71 of these polysaccharides to inhibit bacte- 72 rial adhesion and subsequent biofilm for- 73 mation has been proposed to be a key 74 competitive strategy to allow a producer 75 species to occupy a given environmental 76 niche [5].

77

The structural variety seen in these 78 antibiofilm polysaccharides is diverse, 79 ranging from monosaccharide to hetero- 80 polysaccahride polymers, with no consis- 81 tent feature linked to antibiofilm activity. 82 Both exopolysaccharides and capsular 83 polysaccharides have been identified with 84 antibiofilm activity [6]. Most antibiofilm 85 polysaccharides identified so far have 86 broad-spectrum activity against both clini- 87 cally relevant Gram-positive and Gram- 88 negative pathogens. Critically, this activity 89 is mediated without impacting growth, 90 ruling this out as a mechanism for their 91 antibiofilm properties. Potential mecha- 92 nisms of action include biomasking, signal 93 disruption, gene expression disruption, 94 


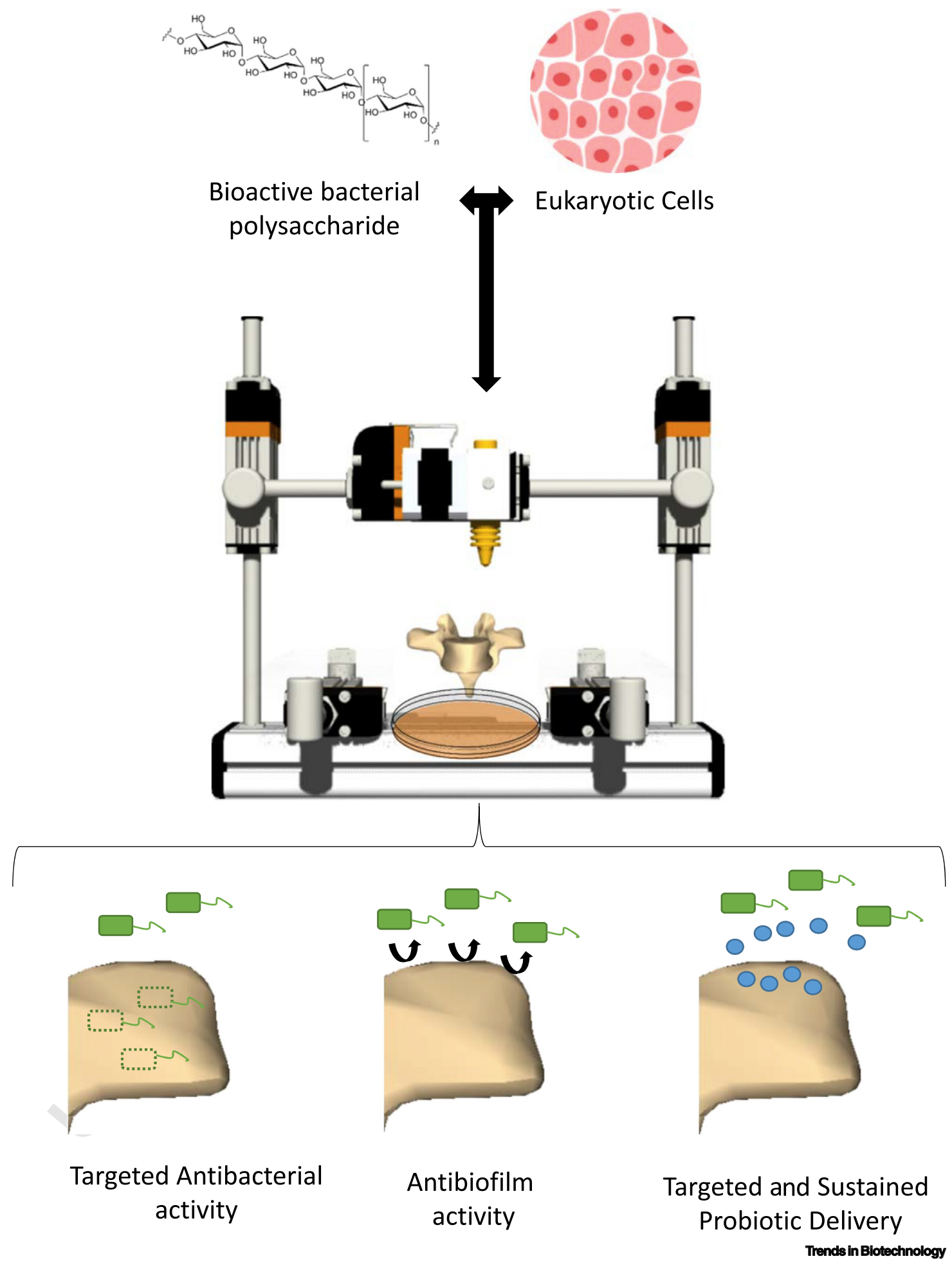

Figure 1. Role of Bioactive Bacterial Polysaccharides in Bioprinting. Functional bacterial polysaccharides can be integrated into current bioink formations to confer antibiofilm or antibacterial activities to 3D bioprinted structures, such as bioprinted bone grafts or prosthetic implants. Integrating these bioactive polysaccharides can reduce the probability of implant reject by preventing bacterial attachment and biofilm formation. Depending on the proposed implantation site, integrating probiotic 
and alteration of biotic/abiotic surface properties [5]. Biomasking is the ability of a bacterial polysaccharide to bind to and occlude native bacterial lectins or sugar-binding proteins that are necessary for biofilm initiation. Indeed, in antibiofilm polysaccharides rich in fucose and fructose, such as EPS1-T14, produced by a marine thermophilic species of Bacillus, biomasking may be a potential mechanism of action because these are known inhibitors of surface lectins [7]. Significantly, several bacterial polysaccharides can disperse already established biofilms. This includes a range of glucose-rich polysaccharides secreted by food-borne lactic acid bacteria and EPS273, a polysaccharide secreted by a marine isolate of Pseudomonas stutzeri. The underlying mechanism of action of this biofilm dispersal activity remains to be uncovered $[8,9]$. However, this suggests that the biomedical applications for such a polysaccharide are not purely prophylactic but could be used to control and disperse established biofilm-associated infections.

Compared with antibiofilm polysaccharides, only a few bacterially derived polysaccharides display antibacterial activity $[9,10]$. ECP, a polysaccharide derived from Enterobacter cloacae, was recently shown to exhibit antibacterial activity against a multidrug-resistant isolate of E. cloacae. While the precise mechanism of action of this polysaccharide remains unclear, it significantly damages the cell membrane [10]. Some of these antibiofilm and antibacterial polysaccharides exhibit further biologically relevant activities, such as antioxidant activity and metal ion chelation activity [8]. Of the anti-infective polysaccharides identified to date, many can also be incorporated into bioinks because of their high levels of thermostability, pseudoplastic rheology, emulsifying activity, and water solubility [3-5]. Critically, most of these anti-infective polysaccharides retain their eukaryotic biological inertness and are considered noncytotoxic $[7,8]$. Given that antibiofilm polysaccharides are nonbiocidal, the capacity for evolved resistance to their activity is significantly diminished. Indeed, in Escherichia coli, resistance to nonbiocidal antibiofilm polysaccharides is rare and requires numerous mutations that significantly alter the surface physiochemical properties of the bacteria [11]. However, the potential for resistance to develop to antibacterial polysaccharides has yet to be explored.

\section{Limitations}

The limited uptake of bacterial polysaccharides as biomaterials is due, at least partly, to costly production methods, difficulty in scalability, and the availability of cheaper synthetic or plant/algal alternatives. However, the emergence of bioprinting has led to an increased interest in bacterial polysaccharides as potential biomaterials for use in a range of medical applications (e.g., wound dressings, tissue regeneration, and bone repair). The capacity for both antibiofilm and antibacterial polysaccharides to be functionally integrated into ink for bioprinting to treat and prevent infection clearly depends on further investigating their biophysical properties. However, they do represent a diverse panel of anti-infective agents that can be used to augment the biocompatibility of traditional bioinks. Rapid advancements in synthetic biology can be utilized to overcome the scalability and production cost issues, whereby the bioactive polysaccharide-synthesising gene clusters can be inserted into synthetic genetic scaffolds to optimize production in workhorse bacteria. This synthetic biology approach may also overcome the issue of minor variations or polysaccharide modifications that can occur in native strains, leading to a loss of homogeneity and potentially bioactivity. The bacteria producing these anti-infective polysaccharides could also be functionally integrated into the bioink itself. Similar methodologies have been used to functionalize a bioink by integrating strains of bacteria capable 172 of degrading pollutants or producing 173 cellulose into already established bioink 174 formulations. These inks can then printed 175 over a given surface in a bespoke geome- 176 try and incubated for a defined period 177 to achieve a desired outcome, such as 178 bioremediation or the formation of a cellu- 179 lose-based synthetic skin scaffold [12]. $\quad 180$

\section{Future Directions}

181

The advent of 4D bioprinting, where the 182 added fourth dimension is the capacity 183 to alter the shape of a 3D printed structure 184 over time or exposure to specific stimuli, 185 also has the potential to transform 186 bioprinting and to have a key role in tack- 187 ling bacterial infections in the future. 188 Hydrogels have already been developed 189 that have shape-morphing capacity [13]. 190 This technology could be used to create 191 programmable wound dressings compris- 192 ing antibiofilm polysaccharides that re- 193 lease antimicrobials upon exposure to the 194 molecular determinants associated with 195 a specific pathogen. However, for this 196 to be implemented, a new mathematical 197 modeling approach is necessary to strate- 198 gically control the sequence of stimulus to 199 act on the stimulus-responsive material 200 and, consequentially, for targeted drug 201 delivery [14]. 3D printed bioinks could 202 also be used as vectors to influence the 203 microbiome. Constructing scaffolds or 204 seeder population reservoirs that can 205 be implanted into locations such as the 206 gut during procedures such as bariatric 207 surgery might pave the way for intelligent 208 microbiota delivery systems. Bioinks 209 need to be regarded not only as a vehicle 210 for cells, but also as being equally im- 211 portant to the cells themselves in terms 212 of biological impact; the drive to use 213 inert polysaccharides will be superseded 214 by the need for polysaccharides with 215 additional bioactivities, such as antibac- 216 terial, antibiofilm, antioxidant, immuno- 217 stimulatory, or metal chelation activity 218 $[8,15]$. Thus, integrating anti-infective poly- 219 saccharides into bioprinting technology 220 
221 has the potential to reduce the incidence

British Council/Newton Fund (2017-RLWKG-17 M.W.U. and G.Y. are supported by National Natural Science Foundation of China (31270150, 51603079, 21774039), China Postdoctoral Science Foundation (2016M602291), and Fundamental Research Funds for Central Universities, Open Research Fund of State Key Laboratory of Polymer Physics and Chemistry, Changchun Institute of Applied Chemistry, Chinese Academy of Sciences.

'Division of Biosciences, Department of Life Sciences, College of Health and Life Sciences, Brunel University London, Uxbridge, UB8 3PH, UK

${ }^{2}$ Department of Biomedical Engineering, Huazhong University of Science and Technology, Wuhan 430074, PR China
${ }^{3}$ Department of Design, College of Engineering, Design and Physical Sciences, Brunel University London, Uxbridge, UB8 $3 \mathrm{PH}, \mathrm{UK}$

\section{${ }^{*}$ Correspondence:}

ronan.mccarthy@brunel.ac.uk (R.R. McCarthy).

${ }^{\circledR}$ Twitter: @mccarthy_ronan (R.R. McCarthy)

https://doi.org/10.1016/.tibtech.2019.05.004

(C) 2019 Elsevier Ltd. All rights reserved.

\section{References}

1. Mandrycky, C. et al. (2016) 3D bioprinting for engineering complex tissues. Biotechnol. Adv. 34, 422-434

2. Lee, J.M. et al. (2018) 3D bioprinting processes: perspective on classification and terminology. Int. J. Bioprint. 4, 151

3. Aljohani, W. et al. (2018) Three-dimensional printing of alginate-gelatin-agar scaffolds using free-form moto assisted microsyringe extrusion system. J. Polym. Res. 25,62

4. Bjarnsholt, T. et al. (2018) Biofilm formation - what we can learn from recent developments. J. Intern. Med. 284, 332-345

5. Rendueles, O. et al. (2013) Antibiofilm polysaccharides. Environ. Microbiol. 15, 334-346

6. Goncalves, M. dos S. et al. (2014) Anti-biofilm activity: a function of Klebsiella pneumoniae capsular polysaccharide. PLoS One 9, e99995

7. Spanò, A. et al. (2016) In vitro antibiofilm activity of an exopolysaccharide from the marine thermophilic Bacillus licheniformis T14. Curr. Microbiol. 72, 518-528
8. Wu, S. et al. (2016) Antibiofilm and anti-infection of a ma- 242 rine bacterial exopolysaccharide against Pseudomonas 243 aeruginosa. Front. Microbiol. 7, 102

9. Abid, Y. et al. (2018) Production and structural character- 245 ization of exopolysaccharides from newly isolated probi- 246 otic lactic acid bacteria. Int. J. Biol. Macromol. 108, 247 719-728

10. Liu, J. et al. (2018) Structural investigation of a poly- 249 saccharide from the mycelium of Enterobacter cloacae 250 and its antibacterial activity against extensively drug- 251 resistant E. cloacae producing SHV-12 extended- 252 spectrum $\beta$-lactamase. Carbohydr. Polym. 195, 253 444-452 254

11. Travier, L. et al. (2013) Escherichia coli resistance to 255 nonbiocidal antibiofilm polysaccharides is rare and medi- 256 ated by multiple mutations leading to surface physico- 257 chemical modifications. Antimicrob. Agents Chemother. 258 57, 3960-3968

12. Schaffner, M. et al. (2017) 3D printing of bacteria into func- 260 tional complex materials. Sci.

13. Kirillova, A. et al. (2017) 4D biofabrication using shape- 262 morphing hydrogels. Adv. Mater. Weinheim 29, 263 1703443

14. Loh, G.H. et al. (2018) An overview of functionally graded 265 additive manufacturing. Addit. Manuf. 23, 34-44 266

15. McCarthy, R.R. et al. (2017) Cyclic-di-GMP regulates 267 lipopolysaccharide modification and contributes to 268 Pseudomonas aeruginosa immune evasion. Nat. Microbiol. 269 2, 17027 